\title{
NUESTRA EXPERIENCIA EN EL TRATAMIENTO DE LA LITIASIS CORALIFORME (1987-2004). REVISIÓN DE LOS RESULTADOS
}

\author{
Victoria Gonzalo Rodríguez, F. Javier Trueba Arguiñarena?', Jesús Rivera Ferro y Ernesto \\ Fernández del Busto.
}

Servicio de Urología y Servicio de Radiología Intervencionista'. Hospital Clínico Universitario de Valladolid. Valladolid. España.

\begin{abstract}
Resumen.- OBJETIVO: Analizar los resultados del tratamiento de la litiasis coraliforme en nuestro servicio atendiendo a los avances tecnológicos que se han producido en los últimos años destacando el papel fundamental que supone el desarrollo de la cirugía endoscópica y de la litotricia extracorpórea por ondas de choque.
\end{abstract}

MÉTODOS: Hemos revisado todos los pacientes tratados de litiasis coraliforme en nuestro servicio entre los años 1987 y 2004.

RESULTADOS: Se consiguió la extracción completa con un sólo acto quirúrgico mediante nefrolitotomía percutánea en un $24.1 \%$. En el $75.9 \%$ restante se realizó un tratamiento combinado mediante litotricia extracorpórea con ondas de choque o una segunda nefrolitotomía percutánea. 09005 Burgos. (España). victoriaCGR@hotmail.com

Trabajo recibido: 4 de febrero 2008 .
CONCLUSIONES: La endourología ha revolucionado el tratamiento de la litiasis coraliforme. Es eficaz sola y en combinación con la LEOC para el manejo de esta patología con un número reducido de complicaciones y una alta tolerabilidad por parte de los pacientes.

Palabras clave: Litiasis coraliforme. Cirugía endoscópica. LEOC.

Summary.- OBJECTIVES: The objective of this work is to analyze the treatment of staghorn calculi at our Department of Urology. We have to know the recent development of endoscopic surgery (percutaneous renal surgery) and external shock wave lithotripsy.

METHODS: We reviewed the surgical management of staghorn calculi during the period between 1987 and 2004.

RESULTS: Percutaneous renal surgery was performed successfully in $24.1 \%$ of the cases. Persisting residual fragments appeared in $75.9 \%$ and were treated by ESWL or second endosco-pic surgery.

CONCLUSIONS: Percutaneous renal surgery may be considered the technique of choice to treat staghorn calculi. Endoscopic surgery has good results and little complications with low morbidity. In other cases the treatment is combined therapy, percutaneous renal surgery and ESWL.

Keywords: Staghorn calculi. Endoscopic surgery. Extracorporeal lithotripsy. 


\section{INTRODUCCIÓN}

La litiasis ha alcanzado tal protagonismo que junto con la patología prostática y las infecciones urinarias conforman los tres procesos más frecuentes atendidos en un Servicio de Urología.

En nuestro país la litiasis renal afecta a un porcentaje de la población superior al $4 \%$ y la recidiva se da en un tercio de los mismos a lo largo de un periodo de cuatro años (1).

La "Enfermedad de la Piedra" como así se la conocía tiene importantes implicaciones médicas, laborales y sociales. Así pues genera importante sufrimiento al paciente en forma de dolor cólico requiriendo atención médica y en ocasiones ingreso hospitalario e inactividad laboral.

En determinados casos la complicación infecciosa $(1,3)$ puede conducir a una sepsis urinaria y a un cuadro de fracaso renal agudo, que requiere una actuación urológica urgente. Por último, el mantenimiento de la infección, la pielonefritis crónica y la uropatía obstructiva pueden conducir a la insuficiencia renal crónica y a la necesidad de instaurar un tratamiento sustitutivo mediante diálisis.

Se estima que de los pacientes que acuden a urgencias con cólico renal y son ingresados el porcentaje oscila entre un $10 \%$ y un $30 \%(1,3)$.

La presencia de cálculos en necropsias junto con le revisión de estudios radiológicos nos proporcionan información de las litiasis "asintomáticas" con porcentajes que oscilan entre el 0.7 y el $5.4 \%$ (2).

La incidencia de la litiasis es muy variable según la zona analizada. En Europa occidental, la incidencia estimada es del $0.5 \%$, con una prevalencia actual del $5 \%$.

En España la urolitiasis tiene una prevalencia del $4.16 \%$ y una incidencia del $0.27 \%$.

En consecuencia se calcula que existen alrededor (2) de 1.600 .000 pacientes con litiasis, con 105.000 casos nuevos por año, de los cuales el $75 \%$ se solucionan espontáneamente (expulsión del cálculo) y el $25 \%$ restante requerirá tratamiento urológico entendiendo como tal la litotricia extracorpórea, la cirugía urológica y la cirugía abierta o combinación de la primera con estos dos últimos procedimientos.

Por otro lado hay que tener en cuenta que la enfermedad litiasica es recidivante (1-3) por definición estimándose una tasa de recidivas del $34.5 \%$.
De ellas sólo un 5-10\% son idiopáticas, en el resto es decir un $90-95 \%$, se detectan alteraciones bioquímicas que podemos controlar cambiando así la historia natural de la enfermedad.

Dentro de los factores epidemiológicos involucrados en la urolitiasis podemos destacar los siguientes (2):

\section{- Edad y sexo}

La incidencia máxima se da entre la tercera y quinta década de la vida. Afecta más al sexo masculino en una relación 2-3/1. En la mujer predominan las litiasis de origen infeccioso (litiasis coraliformes) y las relacionadas con la cistinuria y el hiperparatiroidismo primario, mientras que en el hombre es más frecuente la litiasis de oxalato cálcico y de ácido úrico.

\section{- Raza}

Los sirios, asiáticos e indios tiene mayor predisposición a padecer la enfermedad frente a la raza india norteamericana, la raza negra y los israelitas, quizás por hipovitaminosis D y/o aumento de las sustancias inhibidoras de la cristalización en la orina.

\section{- Herencia}

Es importante en la litiasis de ácido úrico, xantina, y algunos casos de litiasis cálcica secundaria a acidosis tubular renal e hipercalciuria familiar hereditaria.

\section{- Predisposición litogénica}

Debido a alteraciones del metabolismo fosfocalcico (hipercalciuria, hiperoxaluria, hipercistinuria...etc.)

\section{- Dieta}

Se han relacionado numerosos factores dietéticos como productos lácteos, el oxalato, la vitamina $C$, azucares refinados, purinas, proteínas de origen animal, baja ingesta de vitamina B6. De esta manera la dieta vegetariana produce una menor excreción de calcio, ácido oxálico y ácido úrico en la orina disminuyendo por tanto la litiasis urinaria y su recidiva.

\section{- Clima}

Existe una mayor incidencia de litiasis en los meses calurosos debido a una menor ingesta de líquidos.

\section{- Profesión y nivel socioeconómico}

El nivel socioeconómico alto se asocia con dietas ricas en hidratos de carbono refinados, proteínas animales...etc. que aumentan el riesgo litiásico. 


\section{CLASIFICACIÓN DE LAS LITIASIS}

Los estudios cristalográficos de la segunda mitad del siglo XX han permitido el conocimiento de la composición del cálculo y de las estructuras cristalinas de sus diversos componentes, dando origen a la siguiente clasificación: ampliamente difundida $(2,3)$.

\section{- Oxalatos cálcicos:}

- Oxalato cálcico dihidratado y monohidratado.

\section{- Fosfato cálcico}

\section{- Ácido úrico}

- Litiasis "infectiva":

- Fosfato amónico magnésico (estruvita), urato amónico.

\section{- Litiasis de cistina}

- Litiasis de compuestos poco frecuentes:

- Metabólicas: Xantina, ácido orótico

- Medicamentosas: Sulfamidas, triamterene

\section{CLÍNICA}

Los cálculos del aparato urinario se comportan como cuerpos extraños dando lugar a un cuadro clínico variable dependiendo de la localización de la litiasis y de la repercusión anatómica y funcional que produzca sobre el riñón y la vía excretora, bien a través de un efecto irritativo sobre el urotelio o bien por obstrucción urinaria $(1,3)$.

Los síntomas (4) más frecuentes son el dolor tipo cólico renal, el síndrome miccional y la hematuria. Si el cuadro se mantiene pueden producirse complicaciones como la obstrucción de la vía urinaria y/ o anuria e infección urinaria que puede evolucionar a una pielonefritis, pionefrosis ó en casos graves a una sepsis. A largo plazo la persistencia de la litiasis en el riñón, y sobre todo si se trata de cálculos infectivos como es la litiasis coraliforme, que será posteriormente analizadas a lo largo del trabajo, genera un deterioro progresivo de la unidad renal desde el punto de vista tanto anatómico como funcional pudiendo llegar a una anulación total del riñón.

\section{OBJETIVOS}

En el presente trabajo se ha planteado el estudio sobre el tratamiento de la litiasis coraliforme, atendiendo a los avances tecnológicos que se han producido en los últimos años. Destacamos el papel fundamental que supone el desarrollo de la cirugía endourológica y de la litotricia extracorpórea por ondas de choque junto con la tradicional cirugía abierta para el manejo de esta patología.

El objetivo último de este trabajo es el intentar establecer unas pautas de actuación atendiendo al tipo de litiasis coraliforme, combinando el arsenal terapéutico con el que contamos de la forma más eficaz y con la menor morbilidad posible.

\section{MATERIAL Y MÉTODOS}

Hemos realizado un estudio retrospectivo analizando las historias clínicas de 70 pacientes tratados de litiasis coraliforme en el Servicio de Urología del Hospital Clínico Universitario de Valladolid en el periodo de tiempo comprendido entre mayo de 1987 a mayo de 2004.

La mayoría de los pacientes consultaron por un cuadro de cólico renal de repetición acudiendo al Servicio de Urología ambulatoriamente o a través del Servicio de Urgencias.

El diagnóstico de la litiasis se llevó a cabo en todos los casos mediante le realización de una urografía con contraste radiológico. Mediante esta técnica de imagen clasificamos los cálculos en litiasis coraliforme grupo 1, grupo 2, grupo 3 y grupo 4, litiasis pseudocoraliforme y litiasis coraliforme simple también llamada litiasis pielocalicial.

Previo al tratamiento todos los pacientes son sometidos a una serie de estudios preoperatorios que incluyen analítica completa, coagulación, radiografía de tórax, electrocardiograma y urocultivo, este último se realiza para valorar la necesidad previa de tratamiento antibiótico.

Posteriormente se analiza todo el procedimiento quirúrgico haciendo especial hincapié en la vía de abordaje renal atendiendo a la localización de la litiasis. Además valoramos el tiempo de postoperatorio, las complicaciones que han surgido y el manejo de las mismas.

Pasados uno o dos meses los pacientes son sometidos a una nueva urografía para valorar el resultado y plantear un segundo escalón de tratamiento en los casos en que fuera necesario.

Las variables que han sido estudiadas y que posteriormente serán expuestas, fueron analizadas mediante tablas y gráficos utilizando los programas SPSS versión 12.0 y Microsoft Excel 2000. 


\section{RESULTADOS}

Los cálculos coraliformes se denominan así porque ocupan la mayor parte del sistema colector y se asemejan al coral marino, o también a las astas de un venado. Habitualmente forman una masa litiasica renal que ocupa el infundíbulo, la mayoría de los cálices y la pelvis en bloque o bien de manera articulada (Figura 1).

En la mayoría de las ocasiones estos cálculos están compuestos de fosfato amonico magnésico (estruvita), además de materia orgánica, fosfato cálcico o urato amónico entre otros. Un hecho que es importante destacar es que habitualmente se forman en el contexto de infecciones urinarias producidas por gérmenes ureolíticos (Proteus, Pseudomonas, Ureoplasma...)

Según el volumen y la distribución pielocalicial, se denominan coraliforme completo, incompleto o simple (5).

Los coraliformes completos son aquellos que ocupan la pelvis renal y los tres grupos infundibulocaliciales con un volumen superior al $80 \%$ de la vía excretora intrarrenal.

Los cálculos incompletos o pseudocoraliformes se extienden por la pelvis y dos grupos infundibulocaliciales o por los tres con un volumen inferior al $80 \%$ de las cavidades renales.

Los cálculos coraliformes simples o pielocaliciales, son los que ocupan la pelvis y solo un grupo infundibulocalicial.

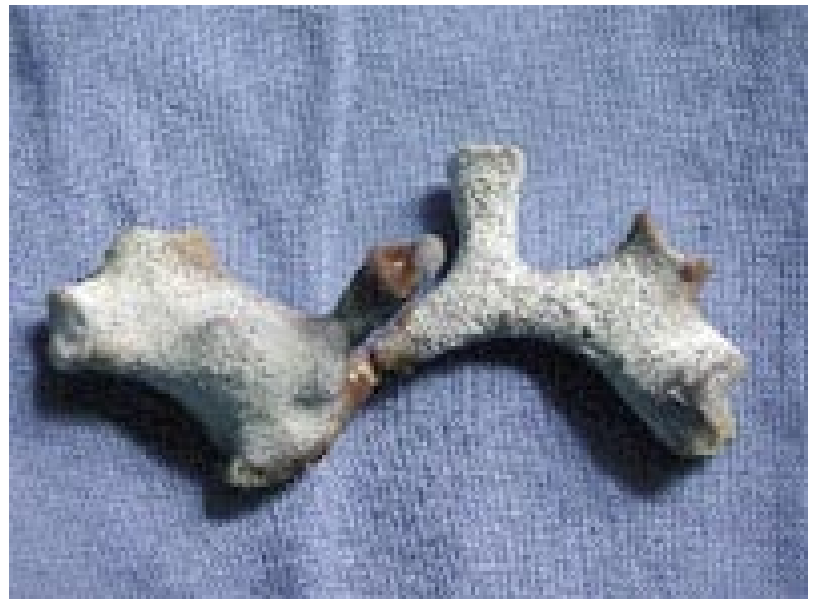

FIGURA 1. Litiasis coraliforme.
Los criterios que se emplean para clasificar las litiasis son (M. Arrabal y cols., 1990): Las características del cálculo (localización, forma, tamaño, consistencia y número), la morfología de la vía excretora y la funcionalidad del parénquima renal.

Atendiendo a estos criterios se establece una clasificación de las litiasis coraliformes en cuatro grupos publicada en 1994 por Segura, J. W. y cols., en The Journal of Urology (Tabla I).

Entre 1987 y 2004 fueron intervenidos quirurgicamente 70 pacientes que presentaban litiasis coraliforme. De ellos 52 casos correspondían al sexo femenino $(74.29 \%)$ y 18 al sexo masculino $(25.71 \%)$. La media de edad de los pacientes fue de 56.08 años, siendo el rango de edad de 23 a 80 años.

El diagnóstico de la litiasis se realizó en todos los enfermos mediante una urografía. A través de esta técnica de imagen observamos que del total de litiasis $43(61.4 \%)$ se localizaban en el riñón izquierdo y $27(38.6 \%)$ en el riñón derecho y sólo en 5 casos el problema era bilateral.

Se obtuvieron 18 casos de litiasis coraliforme simple o pielocalical $(25.7 \%), 17$ de litiasis pseudocoraliforme $(24.3 \%), 5$ casos de litiasis coraliforme del grupo $1(7.1 \%), 9$ pertenecientes a las litiasis coraliformes del grupo $2(12.9 \%), 14$ casos del grupo $3(20 \%)$ y finalmente 7 casos de litiasis coraliforme del grupo 4 (10\%) (Figura 2).

Dentro de las litiasis pseudocoraliformes la combinación de cálices afectados más frecuente ha sido la de cáliz medio con cáliz inferior $(70.5 \%)$. Por su parte dentro del grupo de las litiasis coraliformes simples el cáliz más frecuentemente ocupado por cálculos ha sido el cáliz inferior (72.2\%).

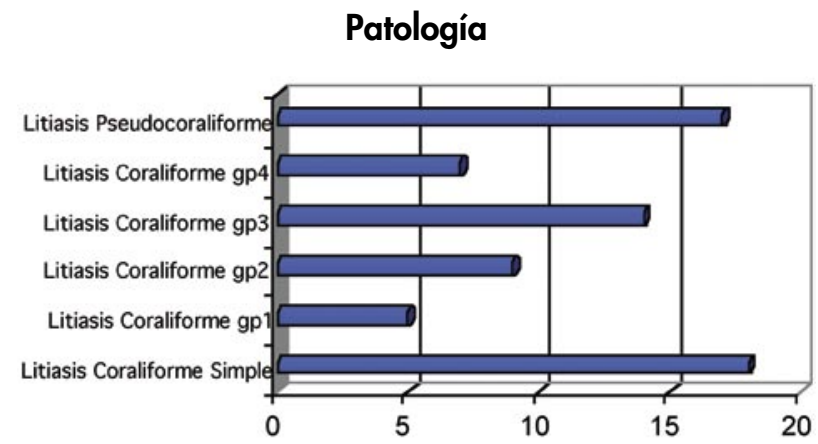

FIGURA 2. Tipos de litiasis coraliformes. 
Todos los pacientes fueron tratados quirurgicamente. El acto quirúrgico se realizó de una forma programada, sin embargo en 5 casos $(7.81 \%)$ fue necesario la colocación de una sonda de nefrostomia percutánea (SNP) de forma urgente (previa a la cirugía) por existir una uropatía obstructiva completa.

En 66 casos se realizó cirugía endourológica mediante nefrolitotomía percutánea (NLP) $(95.7 \%)$, reservándose la cirugía abierta para 4 casos $(4.3 \%)$, en los que se optó por la nefrectomía en 3 de ellos al existir anulación funcional completa del riñón y una pielotomia ampliada en el cuarto caso. Los pacientes en los que se realizó la nefrectomía presentaban litiasis coraliformes del grupo 4 y la pielotomía se realizó para tratar una litiasis coraliforme del grupo 1 de una paciente joven de 27 años (Figura 3).

La vía de bordaje renal más utilizada fue el cáliz postero-inferior, solamente en 10 pacientes hubo que recurrir a un segundo trayecto con el fin de extraer la mayor parte posible de la masa litiasica.

La litofragmentación se realizó en 30 casos mediante litotricia neumática con lithoclast (63.8\%), en 16 pacientes con ultrasónica (34\%) y sólo en 1 caso se empleo litotricia mecánica (2.1\%) (Figura 4). En todos los pacientes se dejó el catéter de nefrostomia durante una media de 3.1 días, realizándose un control radiológico previo a su retirada. Además en 12 casos se colocó un catéter doble J, en unos casos para solventar las complicaciones surgidas y en otros para ser posteriormente enviados a LEOC.

Las complicaciones más frecuentes que se produjeron bien en el acto quirúrgico o bien en el postoperatorio fueron hemorragia que se produjo en 7 casos por punción y manipulación renal $(24.1 \%)$, perforación renal o ureteral en 5 pacientes $(17.2 \%)$, pielonefritis en 2 ocasiones (6.9\%), 2 casos de sep-

\section{TABLA I. CLASIFICACIÓN DE LA LITIASIS CORALIFORME.}

\section{LITIASIS CORALIFORME GRUPO 1}

Superficie litiasica $<700 \mathrm{~mm}^{2}$ (blandos) $/<450 \mathrm{~mm}^{2}$ (duros)

Distribución pielocalicial homogénea

Infundíbulos y cálices poco ramificados

Parenquima conservado

\section{LITIASIS CORALIFORME GRUPO 2}

Superficie litiasica $<700 \mathrm{~mm}^{2}$

Distribución pielocalicial central

Infundíbulos y cálices amplios y dilatados

Parenquima conservado

\section{LITIASIS CORALIFORME GRUPO 3}

Superficie litiasica $>700 \mathrm{~mm}^{2}$ (blandos) $/>450 \mathrm{~mm}^{2}$ (duros)

Distribución pielocalicial central

Infundíbulos y cálices muy ramificados

Atrofia parenquimatosa

\section{LITIASIS CORALIFORME GRUPO 4}

Superficie litiasica $>700 \mathrm{~mm}^{2}$ de cualquier consistencia

Distribución pielocalicial periférica

Infundíbulos estrechos y secuestros caliciales

Atrofia parenquimatosa 


\section{Tipo de intervención}

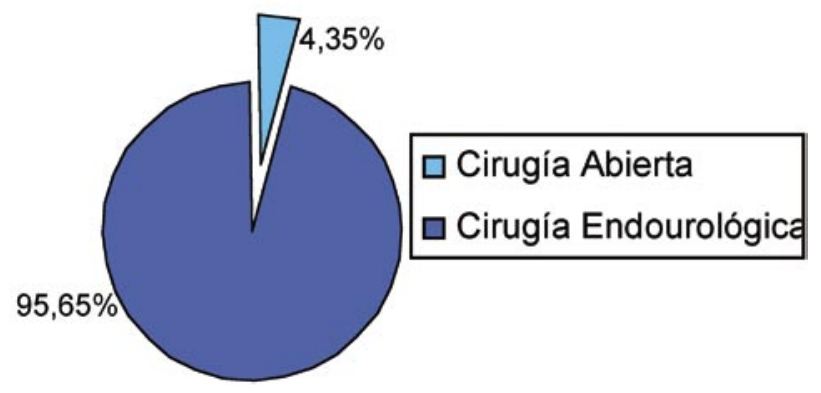

FIGURA 3. Tipo de cirugía realizada.
Cirugía endourológica NLP/
Cirugía abierta nefrectomía.

sis urológica $(6.9 \%)$ y en un 13 casos otras complicaciones (44.8\%) (Figuras 5 y 6 ). El manejo de las complicaciones se realizó en el caso de hemorragia mediante transfusión sanguínea, la perforación renal/ureteral mediante la colocación de un catéter doble J durante 1 mes y los cuadros de pielonefritis y sepsis urinaria con antibioterapia sólo requiriendo un paciente el ingreso en UVI.

El tiempo de postoperatorio presentó una media de 7.78 días, estando el rango entre 3 y 30 días. Los pacientes que logicamente permanecieron más tiempo ingresados fueron aquellos que desarrollaron alguna de las complicaciones ya descritas.

El resultado de la cirugía endourológica mediante nefrolitotomia percutánea a la que fueron sometidos los pacientes, se evalúa en el propio acto quirúrgico mediante el empleo de nefroscopia peroperatoria y con la realización de una nueva urografía al mes o dos meses valorando la existencia o no de restos litiásicos. De esta manera se consiguió la extracción completa con un sólo acto quirúrgico me-

\section{Tipo de litotricia}

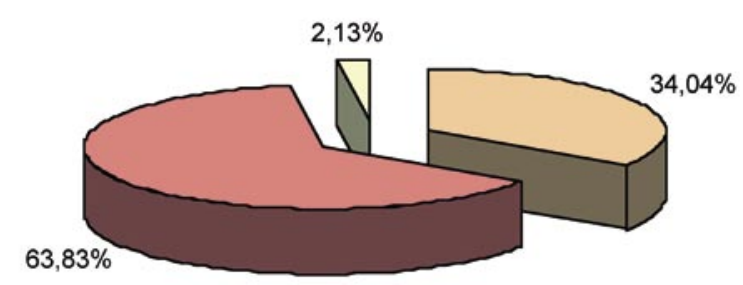

$\square$ Ultrasonidos $\square$ Neumática litoclast $\square$ Mecánica

\section{FIGURA 4. Tipos de litotricia empleada para la fragmentación de las litiasis.}

diante NLP en un $24.1 \%$, quedando restos litiasicos en el $75.9 \%$ (Figura 7 ).

De los pacientes en lo que se consiguió le extracción completa 3 casos eran litiasis coraliformes simples, 4 correspondían con litiasis pseudocoraliformes, 2 casos litiasis coraliformes del grupo 1, 3 del grupo 2 y finalmente sólo un caso del grupo 3 .

En aquellos enfermos en los que no fue posible eliminar toda la masa litiasica lo que se busca con la nefrolitotomia percutánea es realizar una "cirugía litorreductora", que implica la aceptación de restos conocidos en aras de preservar el parénquima renal para un ulterior tratamiento complementario bien con litotricia extracorpórea con ondas de choque (LEOC) ó mediante una segunda cirugía percutánea.

De los pacientes en los que no se consigue una extracción completa existen 12 casos en los que los restos litiasicos fueron mínimos. De ellos 6 correspondían a litiasis coraliformes simples, 4 a litiasis coraliformes del grupo 1, un caso del grupo 2 y el

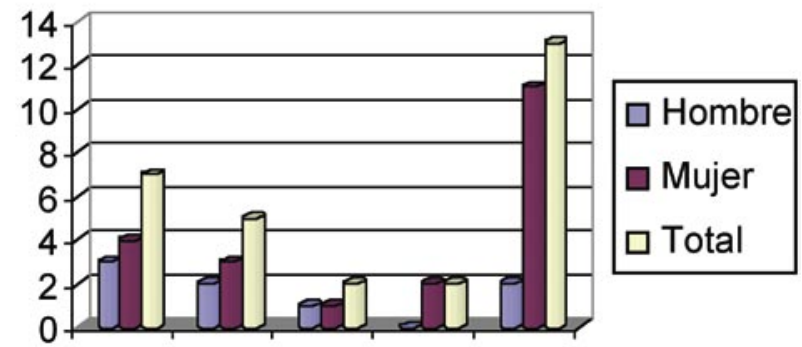

FIGURA 5. Complicaciones secundarias a la cirugía según el sexo.

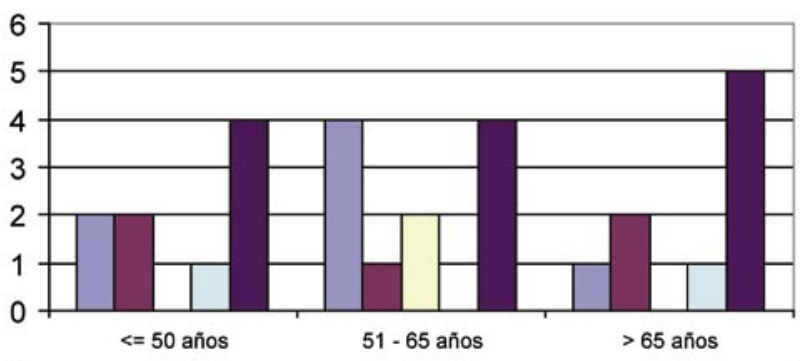

$\square$ Hemorragia $\square$ Perforación $\square$ Pielonefritis $\square$ Shock Séptico $\square$ Otros rangos de edad.

FIGURA 6. Complicaciones quirúrgicas según los 


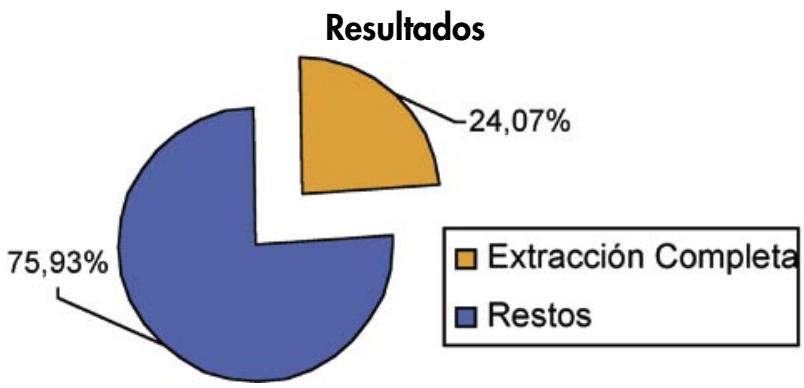

FIGURA 7. Resultados de la cirugía endourológica (un sólo acto quirúrgico) en el tratamiento de la litiasis coraliforme.

ultimo paciente presentaba un cálculo pseudocoraliforme. Estos pacientes están en seguimiento urológico a nivel ambulatorio, y dado que su estado clinico es asintomático y presentan urocultivos negativos no han requerido un segundo escalón de tratamiento.

Existen además 3 casos en los que se aplica una segunda NLP consiguiendo una limpieza completa (dos de ellos litiasis coraliformes del grupo 4 y uno del grupo 3).

Dos casos (litiasis pseudocoraliformes) reciben LEOC y posteriormente son sometidos a segunda cirugía percutánea obteniéndose también una extracción completa.

El resto son sometidos a LEOC siendo muchos de ellos portadores de un catéter doble J para la aplicación de este tratamiento.

Tras la realización de un seguimiento mínimo de un año se recogen dos casos, que tras ser sometidos a tratamiento combinado con NLP + LEOC, presentan anulación funcional renal por pielonefritis crónica, decidiéndose la realización de una nefrectomía, en el resto de los pacientes se consigue la eliminación completa de los restos litiasicos.

\section{DISCUSIÓN}

Revisando la literatura (6-17) podemos encontrar multitud de artículos publicados que tratan el tema de la litiasis coraliforme. Existe una tendencia progresiva a la combinación e indicación electiva de los métodos en función de las características del cálculo. Se mantiene la indicación de monoterapia con litotricia extracorpórea y aumentan las indicaciones de cirugía renal percutánea, sola o asociada a LEOC.
De la revisión de los distintos artículos, no obtenemos unas directrices claras de aplicación clínica pues existen importantes diferencias en los criterios de inclusión, debido a que muchos autores no detallan las características del cálculo (tamaño, composición...) ni la morfología de la vía excretora (6-8).

Yamaguchi, A. (9) refiere un $70 \%$ de buenos resultados con monoterapia de litotricia extracorpórea en cálculos coraliformes de superficie equivalente a $1290 \mathrm{~mm}^{2}$, permitiéndose fragmentos residuales de $100 \mathrm{~mm}^{2}$

Murray, M. J. y cols. $(10,11)$ obtienen un $27 \%$ de buenos resultados solo monoterapia con LEOC, con un $60 \%$ de unidades renales libres cuando la litiasis coraliforme es menor de $500 \mathrm{~mm}^{2}$ y sólo un $8 \%$ si son mayores de $1000 \mathrm{~mm}^{2}$, sin embargo estos autores optan por una NLP como primera poción en los grandes cálculos coraliformes.

Konya, E. y cols. (10) recomiendan terapia combinada NLP + LEOC, en grandes litiasis coraliformes y en su experiencia obtienen resultados similares a los de la cirugía abierta.

Lam, H. S. y cols. $(10,13)$, publican un estudio comparativo entre el empleo combinado NLP + LEOC frente a LEOC, concluyendo la clara superioridad del tratamiento combinado, reservando la LEOC como monoterapia las litiasis de menos de $500 \mathrm{~mm}^{2}$.

En los artículos más recientes, con el desarrollo del ureterorrenoscopio flexible y el láser de Holmio-Yag, observamos como nueva posibilidad terapéutica de las litiasis coraliformes la combinación de la ureteroscopia retrógrada con láser y la LEOC.

La cirugía abierta ha sido durante años el método más empleado para el manejo de las litiasis coraliformes siendo la técnica más adecuada la vía intrasinusal de Gil Vernet, hasta la aparición de las otras técnicas menos invasivas. Así destacamos la tasa de éxito conseguida por Gil Vernet y Ballesteros en el tratamiento quirúrgico de 198 casos de cálculos coraliformes completos operados entre 1979 y 1988 que fue del $98.5 \%$.

Por su parte Boronat, F. y cols. (12) obtiene un $31 \%$ de unidades renales libres de litiasis tras aplicar cirugía abierta y LEOC en un segundo tiempo, con una media de 2.5 actos terapeúticos por paciente.

El éxito obtenido por la litotricia extracorpórea por ondas de choque en el tratamiento de las 
litiasis renales de pequeño tamaño, abre el camino de su aplicación a la litiasis coraliforme asumiendo que requieren un mayor número de sesiones.

Orsola, A. y cols. (10) recomiendan a opción del empleo de la LEOC para litiasis coraliformes de los niños.

Boronat (12), Arrabal y cols. obtienen un tratamiento completo mediante LEOC del $40.8 \%-52.6 \%$, siendo del $66-80.5 \%$ al combinarla con la NLP.

La cirugía renal percutánea $(14,15)$ tiene actualmente como principal indicación la litiasis coraliforme, ya sea en monoterapia o combinada con otras técnicas.

En lo que respecta al abordaje renal (14), se prefiere que la vía sea única por cáliz posteroinferior como hemos realizado en todos nuestros pacientes. La litotricia de contacto con lithoclast y ultrasonidos ha sido efectiva en el $80 \%$ de los cálculos de consistencia dura.

Nguyen, H.C. y cols. en su experiencia con 140 casos de litiasis coraliformes tratados con terapia combinada NLP + LEOC obtiene un $91 \%$ de unidades renales libres. Por su parte Bianchi, G. y cols., obteniendo una tasa de éxito del $93.2 \%$.

Las complicaciones $(16,17)$ de la NLP en el tratamiento de la litiasis coraliforme varía dependiendo de si se realiza un abordaje único o múltiple (hemorragia, fístula renal, infección, fístula arterio-venosa). A pesar de ello las complicaciones de la NLP (vía única por cáliz inferior) combinada con LEOC, son inferiores que las registradas con monoterapia con LEOC o cirugía abierta.

\section{CONCLUSIONES}

Con el paso de los años lo que se ha intentado es conseguir en primer lugar una correcta clasificación de las litiasis coraliformes con aplicación terapéutica y en segundo lugar determinar que técnica o asociación de las mismas es la más adecuada en cada caso.

Sin embargo no debemos olvidar que sea cual sea la técnica aplicada, el fin primordial que se persigue con el tratamiento de la litiasis es dejar al paciente libre de cálculos con la menor morbilidad posible, un coste razonable y una reincorporación a la vida cotidiana en el menor tiempo posible. A pesar del gran arsenal terapéutico a nuestra disposición la consideración más importante es la de hacer una in- dicación correcta de acuerdo con el tipo de cálculo a tratar, la vía urinaria que lo alberga y el contexto clínico del paciente.

Una vez analizados nuestros resultados a lo largo de estos años consideramos que la NLP como monoterapia nos ha permitido la extracción completa de la litiasis en un $25 \%$ con una baja tasa de complicaciones y una buena tolerabilidad por parte de nuestros pacientes. En el resto de los casos debemos realizar una reducción de la masa litiásica para posteriormente aplicar un segundo escalón de tratamiento. La combinación idónea de las técnicas va a depender del tipo de litiasis coraliforme a tratar así como de la tecnología de la que dispongamos:

\section{Litiasis Coraliforme Grupo 1:}

- NLP

- LEOC

- URS + LEOC

Litiasis Coraliforme Grupo 2:

$-N L P$ máticos)

- NLP + LEOC (si fragmentos residuales sinto-

Litiasis Coraliforme Grupo 3:

$-N L P+L E O C$

\section{Litiasis Coraliforme Grupo 4:}

- Cirugía abierta

- Nefrectomía si existe anulación renal

\section{BIBLIOGRAFÍA Y LECTURAS RECOMENDADAS ( ${ }^{*}$ lectura de interés $y^{* *}$ lectura fundamental)}

1. RESEL ESTÉVEZ, L.: "Urología: Libro del Residente". Asociación Española De Urología. ENE publicidad S.A, 471, 1998.

*2. GMS Urología.: "Litiasis urinaria". Grupo Masson, Editorial Garsi, S.A. 5, 1999.

*3. FERNÁNDEZ DEL BUSTO, E.: "Manual de Urología". Secretariado de Publicaciones. Universidad de Valladolid, 233, 1993.

4. DÍAZ BERROCAL, J.; GUILLERMO CATAÑO, J.; MIGUEL SILVA, J. y cols.: "Guía de Manejo de la Litiasis Renal Basadas en la Evidencia". Sociedad Colombiana de Urología, 2001.

5. ARRABAL MARTÍN, M.; REINA RUIZ, M.C.; LANCINA MARTÍN, J.A. y cols.: "Clasificación clínico-terapéutica de la litiasis urinaria". Arch. Esp. Urol., 45: 661, 1992. 
6. CLAYMAN, R.V.: "Combined treatmentof staghorn calculi by fiberoptic transurethral nephrolithotripsy and extracorporeal shock wave lithotripsy”. J. Urol., 162: 1904, 1999.

7. GUNTHER, E.; MARCOTI, M.: "Resultados de la litotricia Extracorpórea en el Hospital Regional de Iquique". Revista Chilena de Urología, 67: 165, 2002.

8. OHSHIMA, S.; ONO, Y.; SAHASHI, M. y cols.: "Extracorporeal shock wave lithotrpsy using the Donier MPL 9000 lithotriptor”. Urol. Int., 52: 17, 1994.

9. YAMAGUCHI, A.: "Extracorporeal shock wave monotherapyfo staghorn calculi". Eur. Urol. Int., 25: 110, 1994.

**10. ARRABAL MARTÍN, M.; GUTIÉRREZ TEJERO, F.; OCETE MARTÍN, C.: "Tratamiento de la Litiasis Coraliforme”. Arch. Esp. Urol., 57: 9, 2004.

11. ARRABAL MARTÍN, M.; BANNUS GASSOL, J.M.;BORONAT TORMO, F. y cols.: "Tratamiento actual de la litiasis coraliforme. Valoración de la cirugía, técnicas percutáneas y litotricia por ondas de choque". Actas Urol. Esp., 21: 1027, 1997.

12. BORONAT TORMO, F.; PONTONES MORENO, J.L. y cols.: "Tratamiento de la litiasis renal cálcica. LEOC, NLP, cirugía abierta”. Arch. Esp. Urol., 54: 909, 2001.

*13. SAXBY, M.F.; SORAHAN, T.; SLANEY, P. y cols.: "A case control study or percutaneous nephrolithotomy versus extracorporeal shock wave lithotripsy". Br. J. Urol., 79: 317, 1997.

14. FERNÁNDEZ DEL BUSTO, E.; TRUEBA ARGUIÑARENA, F.J.: "Endourología del Tracto Urinario Inferior". Secretariado de Publicaciones. Universidad de Valladolid, 8, 1991.

15. ALKEN, P.; HUTSCHEREITER, R.; GUNTHER, R. y cols.: "Precutaneous stone Manipulation". J. Urol., 125: 463, 1981.

16. RINCÓN RÍOS, F.; ORTIZ HERNÁNDEZ, S.: "Experiencia en el Manejo Quirúrgico de la Litiasis Coraliforme". Revista Venezolana de Urología, 47: 19, 2001.

17. SEGURA, J.W.; PREMINGER, G.M.; ASSIMOS, D.G. y cols.: "Nephrolithiasis clinical guidelines panel summary report on the management of staghorn calculi”. J. Urol., 151: 1648, 1994. 